\title{
Performance Evaluation of Routing Protocols in Manets Deployed for e-Learning Purpose
}

\author{
Fatima Lakrami \\ Department of Physics \\ STIC Laboratory, Chouaib \\ Doukkali University \\ El Jadida, Morocco
}

\author{
Najib Elkamoun \\ Department of Physics \\ STIC Laboratory, Chouaib \\ Doukkali University \\ El Jadida, Morocco
}

\author{
Ouidad Laouidya \\ Department of Physics \\ STIC Laboratory, Chouaib \\ Doukkali University \\ El Jadida, Morocco
}

\begin{abstract}
This paper studies the impact of mobility on adhoc routing protocols in an e-learning environment. Such context must take into account the requirements of the transmission of real-time applications, but also the manner of planning adhoc networks for educational purpose. This work initially targets the planning of the routing part in manets, while taking into account several parameters such as density, mobility, traffic load.... The objective is to design a flexible adhoc architecture suitable for elearning systems, for a deployment in university campuses.
\end{abstract}

\section{Keywords}

Adhoc; routing protocol; e-learning; 802.11

\section{INTRODUCTION}

Ad Hoc networks are characterized by a total lack of centralized Mobile hosts that form themselves the network infrastructure. No assumption or limitation is made on the size of the ad hoc network. A single adhoc network can contain hundreds or thousands of mobile units and all elements must cooperate in order to create a temporary architecture to Facilitate disseminating data from any source to any destination in the network.

There are many advantages of deploying Ad-Hoc network. In fact, they provide access to information and services regardless of the geographic position, so there is no need of any central network administration. Ad hoc networks possess multiple applications, and can replace wired networks in several contexts. They represent nowadays a very active research area; they can be deployed in several applications apart from extending connectivity. In fact, they can find application in many other fields. In the last few years the context of e-learning begins to emerge, and the use of multimedia presentations as a teaching medium is currently considered to be an outline of the interactive trend of future education. Manets can obviously serve such purposes and then be used to cover educational institutions, by establishing connectivity between all involved entities (students and teachers), so several scenarios become possible. An example of students localized at different classrooms are communicating through a large scale network, exchanging data, or attending a live streaming course, in such cases, it could be very beneficial to instantly being able to deploy a large network to achieve this communication. The network become more interesting then deploying several Aps or wired networks to enable nodes to communicate.

In order to make the implication of manets effective in such experiments, a preliminary planning must be done. A Planning that refers to physical and configuration modalities. In this paper, the part of the routing is examined. The goal is to find the best configuration that ensures good transmission performance, for the use of manets in education. A real example is considered and will be studied through several scenarios. The specifications related to the physical configuration will also be taken into account, namely the transmission standard, the frequency band..., in order to clearly define the perimeter of the study. The rest of the paper is organized as follow:

Section 2 is about related works. Section 3 presents a review of routing protocols. Section 4 explains the deployment of ad hoc networks in e-learning systems. Section 5 describes the networks architecture of the educational environment, simulation and results while section 6 concludes de paper.

\section{RELATED WORKS}

Comparing manets routing protocol were the subject of a large number of research in ad hoc field [1][2]. Different results were obtained, due to different assumptions about network topology and configuration.

This work is mainly focus on deploying manets for educational purposes. And specially to cover universities departments. Works on deploying manets for eLearning purposes are very few, and are not generally intended to meet real needs in terms of network coverage. Authors in [3] propose the use of wireless ad hoc networks to provide e-Learning for people in rural and remote areas of Zambia, they gave an overview of the faisability of such proposition without specifying the network configuration. [4] presents a project aiming to design and implement an adaptive infrastructure for an efficient collaboration support in ELearning scenarios which take place in mobile multi-hop ad-hoc networks (MANET). An application of a mobile e-learning network which operates totally decentralised with the help of an underlying ad hoc network architecture is described by [5]. In fact, none of the cited works, describes the specfication of the real deployement of manets, it is still representing a number of assumputions about planning and using such network to accomplish a specific task. The question that remains here, is about the suitable configuration to adopt when implementing such infrastructure in educationnal environments

\section{REVIEW OF MANETS ROUTING PROTCOLS}

The process of Routing is defined in the general context as the method of routing information through a network of Connection. The purpose of routing is to route packets from a source to a destination with optimizations (in terms of certain performance criteria). It is therefore important to find Low-cost investment, which will ensure the routing of traffic and ensure its reliability by case of breakdown that may occur on links or on nodes.

Ad hoc network [6] is an environment where network management is distributed across all network elements, the transmission range of each mobile is limited, so in order to make nodes to communicate, routing algorithms are required, for supporting maintenance and for the reconstruction of temporary 
paths, in addition to their ability to establish efficient routes between a pair of nodes. Due to the limitations of ad hoc networks, the construction of paths must be done with a minimum of control traffic and then less bandwidth and energy consumption. Depending on how a routing protocol creates and maintain its paths when disseminating data, routing protocols can be divided into two large families, each having a different mode of operation, the first family known as reactive prototypes, it relies on the following principle: nodes do not keep a routing table up to date but perform routing on demand. the second family, that of proactive protocols, maintains routing tables (through periodical exchanges of topological messages). Once the routing table is built, the nodes can communicate.

In the following, a brief review of the best-known standardized protocols, proposed for routing in ad hoc networks., is presented.

\subsection{AODV}

AODV (for Ad hoc On Demand Distance Vector) is a routing protocol for mobile networks (ad hoc network). It is capable of both unicast and multicast routing. It is loop-free, self-starting and accommodates a large number of mobile (or intermittent) nodes. When a source node requests a route, it creates routes on the demand and maintains them as long as the source needs them. For multicast groups, AODV constructs a tree structure. This routing protocol is energy intensive and does not require high computing power, so it is easy to install on small mobile devices [7].

\subsection{DSR}

The "Dynamic Source Routing" [8] protocol is based on the use of the "source routing" technique. In this technique, the data source determines the complete sequence of nodes through which the data packets will be sent.

\subsection{OLSR}

OLSR is a proactive routing protocol designed to operate in a distributed mobile environment without any central controlling and mobility-responsive (ad hoc networks) [9]. It is used in dense, low-mobility networks.

It represents an adaptation and optimization of the principle of link state routing for ad hoc networks. It makes it possible to get the roads shorter path. The optimization is due to the fact that in a link state protocol, each node declares its direct links with all its neighbors to the whole network. In the case of OLSR, the nodes will declare only a subset of their neighborhood by the use of multipoint relay MPR (Multipoint Relay).

\subsection{TORA}

The TORA (Temporary-Ordered Routing Algorithm) protocol [10] is a protocol initiated at the source (initiated source), developed primarily for high mobility ad hoc networks. In order to support a number of deletions of links induced by the high mobility of the nodes, several paths potentials, between a source $\mathrm{i}$ and a destination, are calculated. In addition, in order to reduce control traffic resulting from the deletion of a link, this protocol quickly notifies changes to nodes and periodically propagates these changes to the rest of the network, but at a low frequency.

\section{ADHOC IN ELERANING CONCEPT}

Applications of adhoc networks are very numerous and very diverse. In the context of e-learning, it is very interesting to be able to construct spontaneous communication schemes at the basis of manets. For this purpose, several scenarios become possible. An example of groups of students localized at different classrooms, set up an experiment on the basis of a video broadcasted by their teacher, located in a nearby classroom or in his office. In this case, students must be able to follow the video in real time to achieve successfully the experiment or the attended course and also to communicate with each other, asking questions and even searching for further information on the web. This communication architecture must be simple, spontaneous and efficient.

In this work, the main concentration is on the qualitative and quantitative analysis in planning the way of deploying such networks in real contexts. Similar works have already been done in this field considering different constraints and using different simulation scenarios, but without considering real cases.

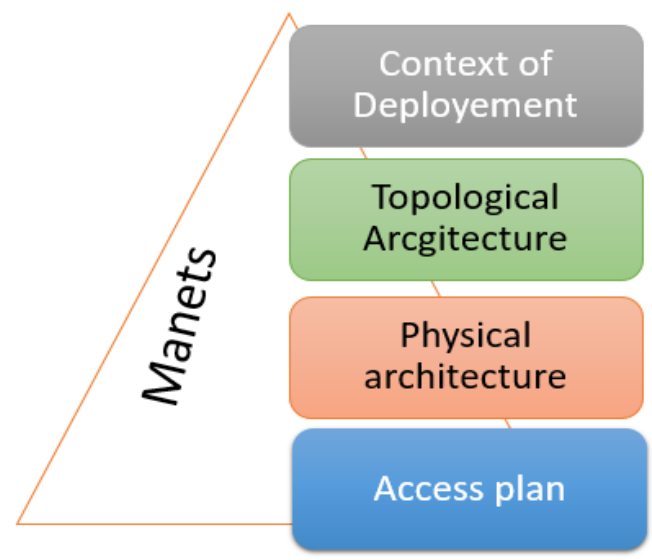

Fig 1: Ad hoc network planning process

Many students, computer scientists, and researchers have come through several works and researches about how to plan a suitable ad hoc architecture, in terms of physical [10] and routing configuration. But it was impossible to generalize the resulting conclusions obtained for the studied cases, about the efficiency of one configuration compared to another. So, at the end, there is considerable amount of mix reaction in support for example for On Demand routing protocols as much as there is considerable amount of other studies that support proactive routing protocols.

\section{SIMULATION AND RESULTS}

\subsection{Network architecture}

This work is a part of a project aiming to design and implement an adaptive infrastructure for an efficient collaboration support in E-Learning scenarios which take place in mobile multi-hop ad-hoc networks (MANET). Therefore, it is necessary to develop special concepts in order to be able to cope with the high dynamics in such networks. Rather than adopting traditional concepts and dealing with the effects of mobile ad-hoc networks. The characteristics of the inherent dynamics will instead be analyzed for possible benefits regarding collaborative ELearning scenarios. Following the notion of spontaneously connecting computer terminals, an efficient infrastructure should suggest potentially useful collaboration partners and resources according to the educational profile of each student or service respectively. Hence, a spontaneous exchange of experience and knowledge should be supported.

To deal with such problematic, the principal of "divide to rule" is adopted. In fact, it is more difficult to make global design of the network at a global level, so our planning adopts the structuration presented by figure 1 . Actually, this works is falls under the design of the topological architecture.

As far as the physical part is concerned, we chose to work with the 802.11n standard which allows to offer very high flows compared to its previous $802.11 \mathrm{~g}$ according to a previous work [10]. The network is evidently spontaneous and aims to create communicating parts between adjacent classes. 


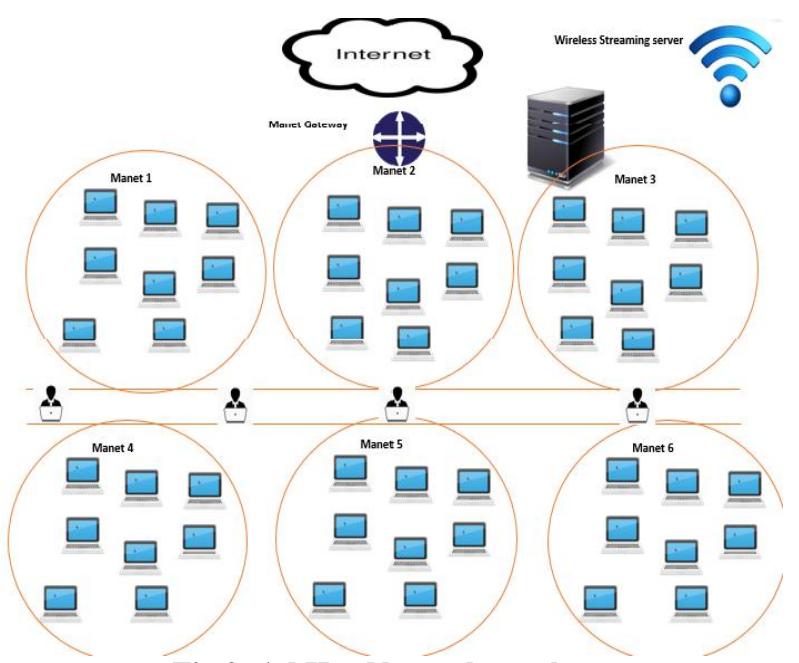

Fig 2: Ad Hoc Network topology

The studied topology is given by figure2. It is composed from 6 classrooms belonging to physical department, each classroom contains at max 10 students with mobile computers, while a number of users are moving through the hall connecting different classrooms. Users are connected to internet through a manet gateway. A gateway is a mobile or stationary communicating device, which works as an interface between different networks to improve their coverage and connectivity. Gateway type, architecture and functionality vary with the different categories of heterogeneous network. In order to form several ad hoc networks, we have to define several sphere of activity of an adhoc network, which must contain groups of students with similar educational objectives. For this purpose, practical work context is chosen to simulation scenarios. In fact, in this case of study, all students participate in the same educational system which mainly supports similar studies.

The participation in the E-Learning system should be supported by several kinds of mobile terminals to provide the opportunity to study any-where and any-time. In this context, different ELearning Profiles are defined. In the proposed E-Learning scenario, we consider a number of participant categories, with mutual interest. Every potential participant has a list of interests which is realized in terms of knowledge, learning and service. On the basis of the collected information's, we developed a local database that enable us to create 5 distinct profiles:

Table 1. Users applications profile's

\begin{tabular}{|c|c|c|c|c|c|}
\cline { 2 - 5 } \multicolumn{1}{c|}{} & $\begin{array}{c}\text { FT } \\
\text { P }\end{array}$ & $\begin{array}{c}\text { EMAI } \\
\text { L }\end{array}$ & $\begin{array}{c}\text { WE } \\
\text { B }\end{array}$ & $\begin{array}{c}\text { DATABAS } \\
\text { E }\end{array}$ & $\begin{array}{c}\text { Vide } \\
\text { Conf } \\
\text { Conf }\end{array}$ \\
\hline Profil_Student_Basic & & & & & $*$ \\
Profil_Student_Advan & $*$ & & $*$ & & $*$ \\
Profil_Researcher & & $*$ & $*$ & $*$ & \\
$\begin{array}{c}\text { Profil_Professor } \\
\text { Profil_Student_Outsi } \\
\text { de }\end{array}$ & $*$ & $*$ & $*$ & & $*$ \\
\hline
\end{tabular}

\subsection{Simulated scenarios}

The following study aims to determine the performance of different routing protocols under the impact of several parameters: mobility, and traffic load. Here, the assumption is that three classes are attending a video of an experiment relative to a physical course, the rest of classes are destined for relaying traffic as a part of the whole adhoc network. the traffic load is defined in function of clients surfing on the web for different reasons: Web, Email, Data...

OPNET 17.5 [12] is used to simulate the current work. The following table describes different characteristics of each impact factor:

Table 2. Traffic and mobility parametrs

\begin{tabular}{|c|l|l|}
\hline & \multicolumn{1}{|c|}{ Traffic } & \multicolumn{1}{c|}{ Mobility } \\
\hline Low & 2 clients & $0 \rightarrow 0.5 \mathrm{~m} / \mathrm{sec}$ \\
Medium & 5 Clients & $0.5 \rightarrow 1.5 \mathrm{~m} / \mathrm{sec}$ \\
High & 10 Clients & $1.5 \rightarrow 3 \mathrm{~m} / \mathrm{sec}$ \\
& & \\
\hline
\end{tabular}

\subsection{Results of simulation}

- Low Traffic load 2 clients

Figures bellow illustrate differents performance metrics obtained in terms of delay loss_delay and packet variation delay for multimedia and best effort traffic.
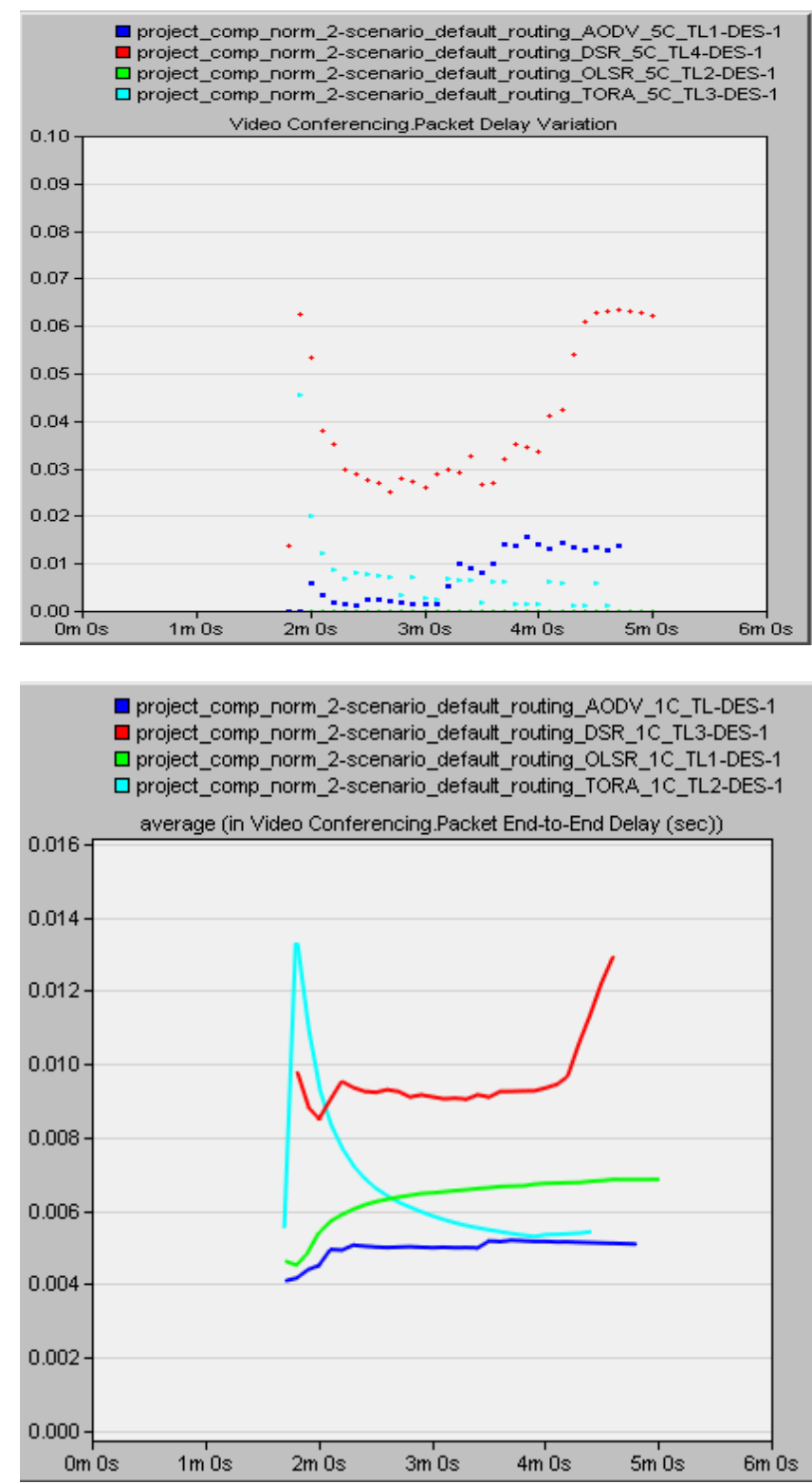


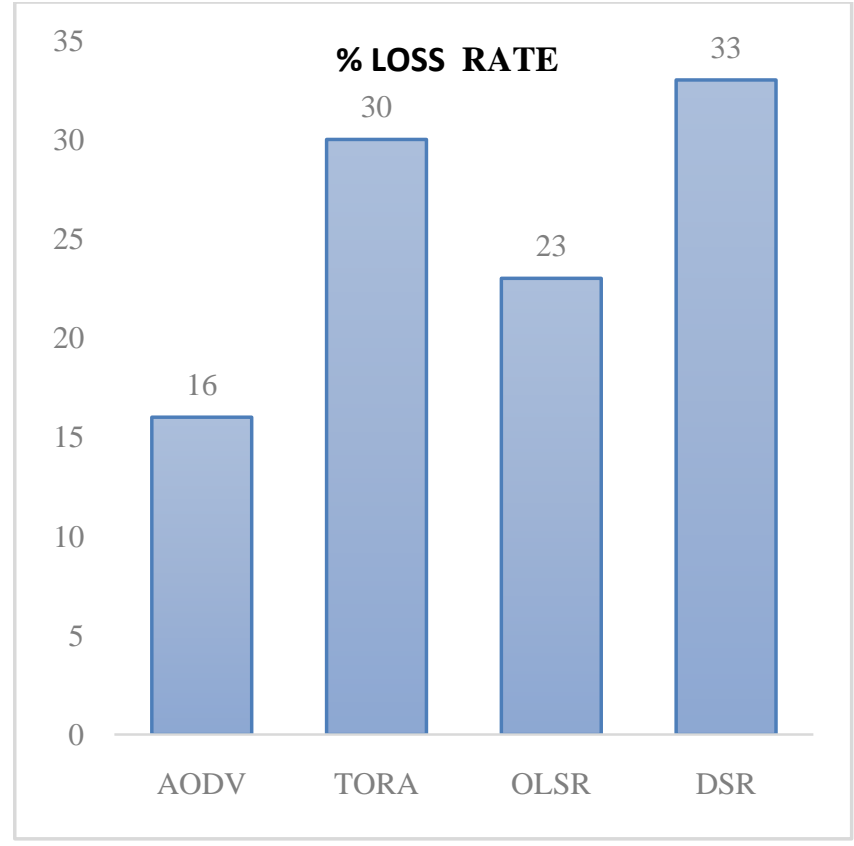

Fig 3: Performance comparison of manet routing protocols for low traffic load

As shown by figure 3 , the minimum delay for transferring the video traffic is obtained for the OLSR and AODV. While the highest value is obtained for DSR. The lowest loss rate is obtained for AODV. This allow to conclude that for this first scenario and by introducing only 2 clients that exchange best effort traffic with the server, it is the AODV protocol that performs better.

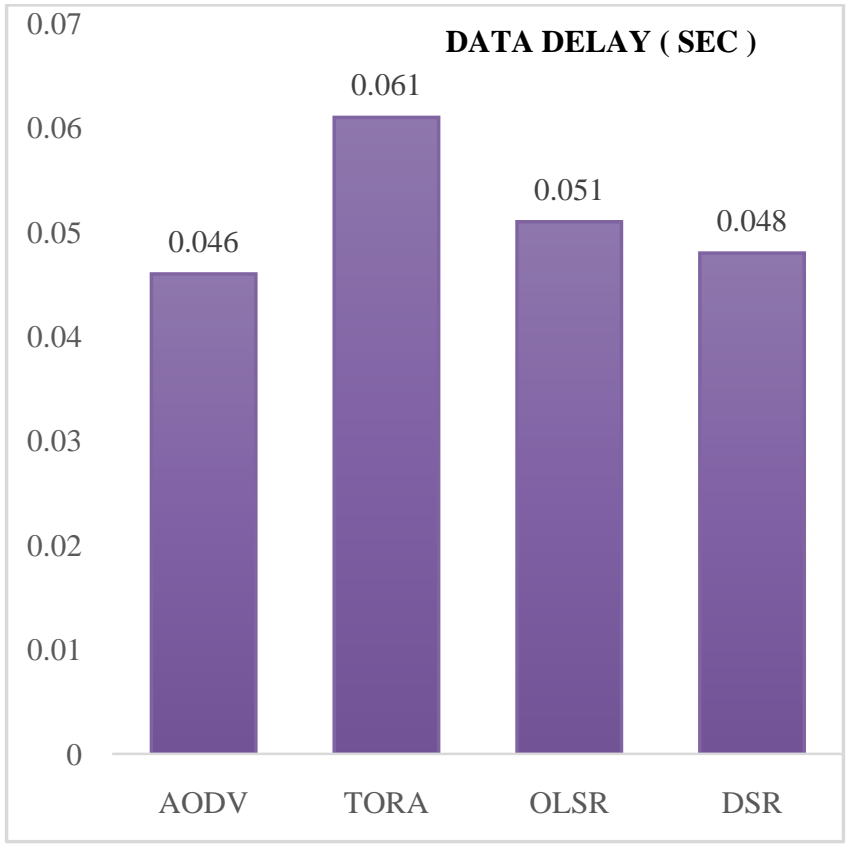

Fig 4: Average Delay (sec) for DATA Traffic

AODV still performs better, even for best effort traffic, this reactive protocol demonstrates higher performance for the considered scenario.

- $\quad$ Medium Traffic Load 5 clients
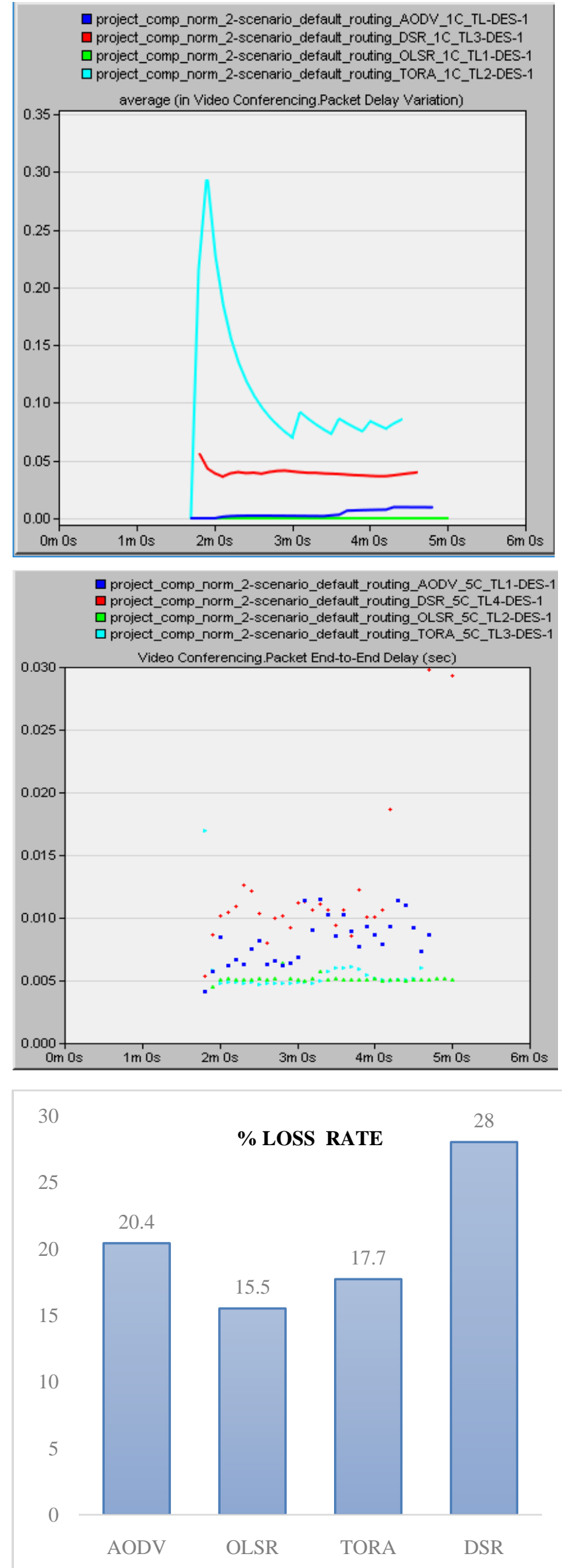


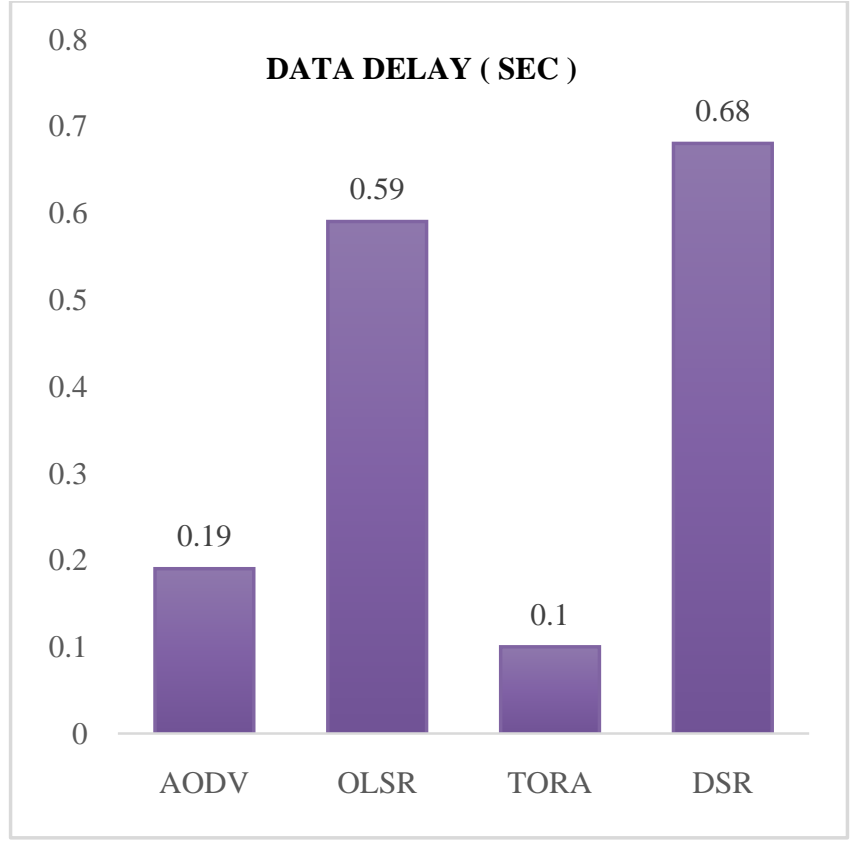

Fig 5: Network performance évaluation with medium traffic load

For this second case, there is a heterogeneity in the obtained results. For multimedia traffic, the best performance is obtained for OLSR (minimum delay and loss rate), while for best effort traffic, it is TORA that performs better.

- $\quad$ High Traffic Load : 10 clients

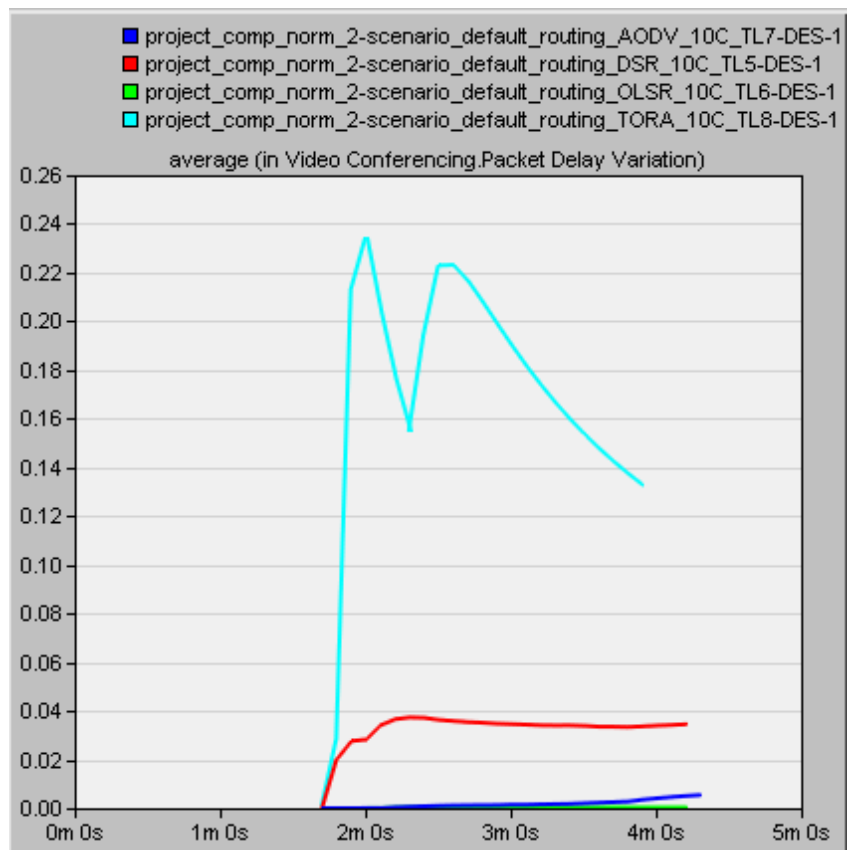

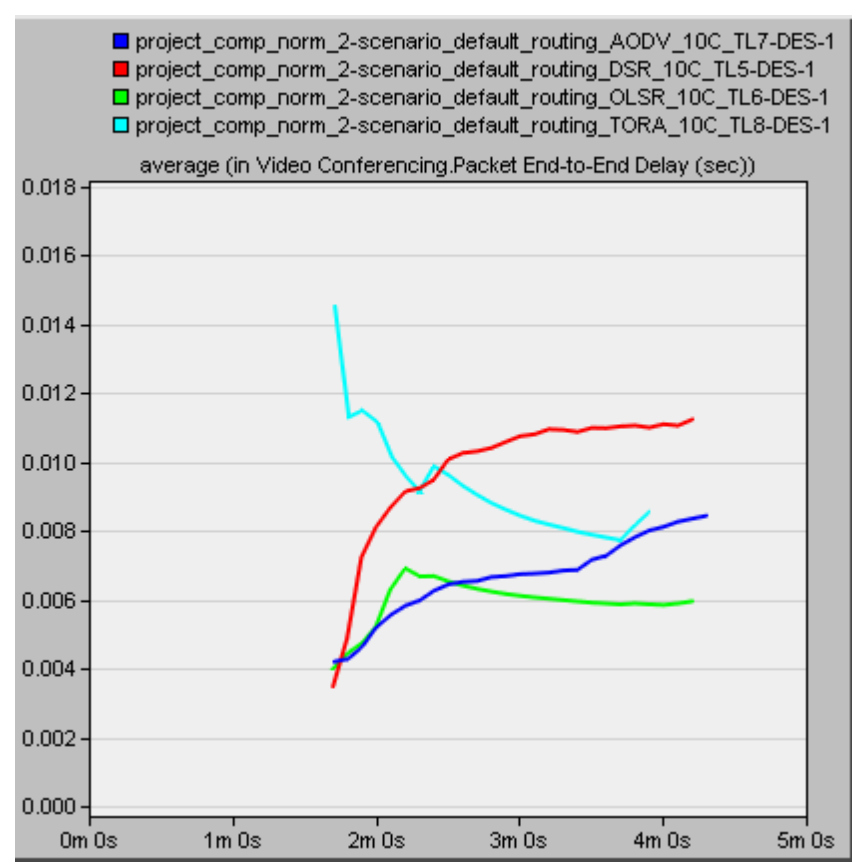

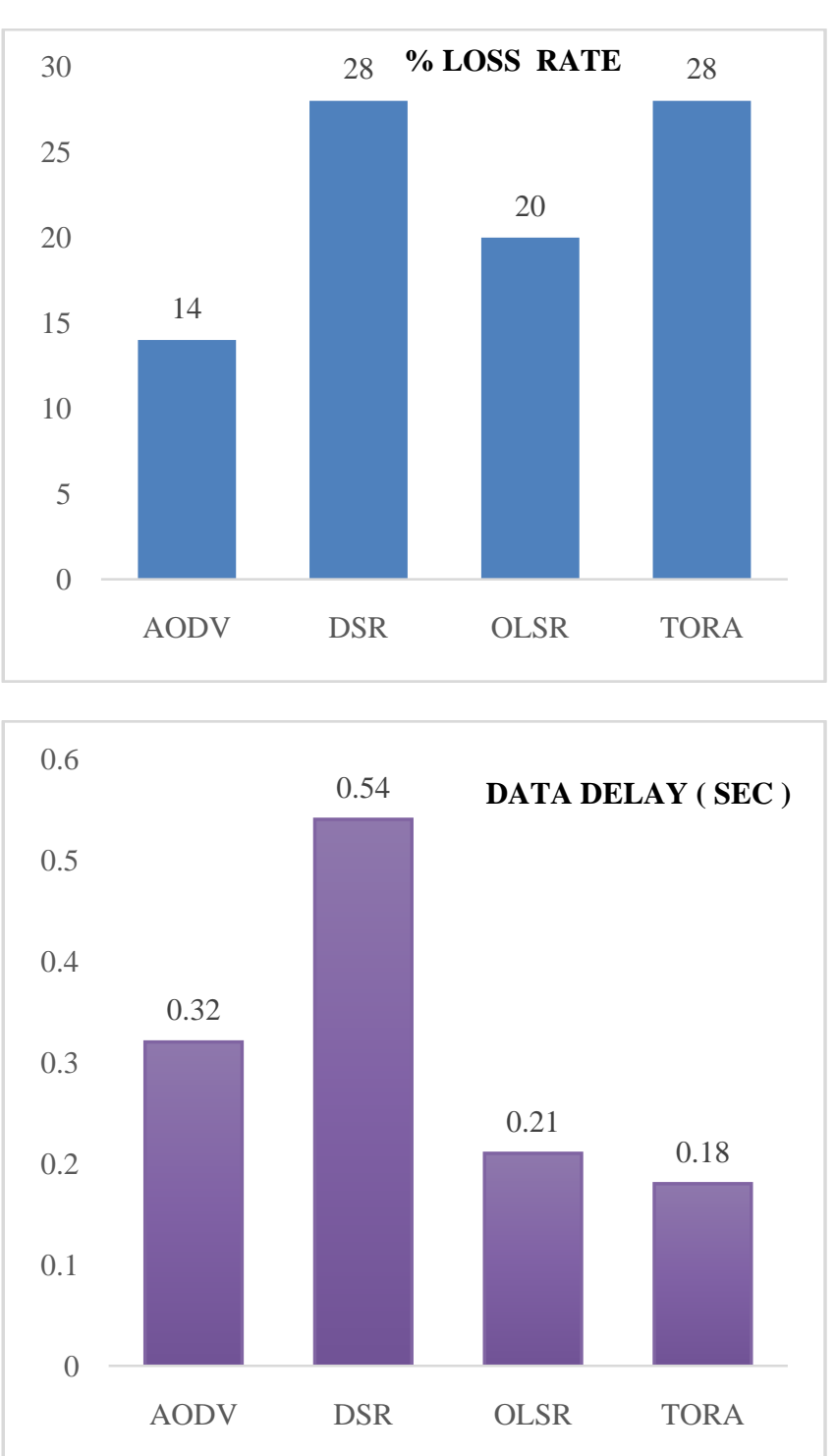

Fig 6: Network performance évaluation with high traffic load 
Results are the same as for 5 clients. OLSR is still the best for multimedia traffic, while TORA shows better results for data traffic.

In fact, OLSR is a proactive protocol, which means, routes are established in advance, and then real traffic have no need to wait until the routing protocol constructs paths to use them. Which assure a certain real time dissemination.

- High Traffic Load : 10 clients with Mobility imact

In MANET, when the node moves from one location to other, the velocity, acceleration, and location of users vary with respect to time. Hence, different mobility models are used to identify these mobility patterns and there is a requirement to analyze various mobility models. The reason is that the mobility model has great impact on the performance of the routing protocol. Otherwise, the performance measures of MANET may not be accurate and it can mislead the application. So, it is necessary and essential requirement to choose the underlying mobility model while evaluating the performance of MANET. In this context of study, the mobility of nodes can be predicted according to a linear trajectory. Users are moving from one classroom to another with a speed of walking. Mobility profiles are defined according to table 2 .

A mobility profile is added to the scenario with high traffic load. Figure7 resumes different results for both video and data:

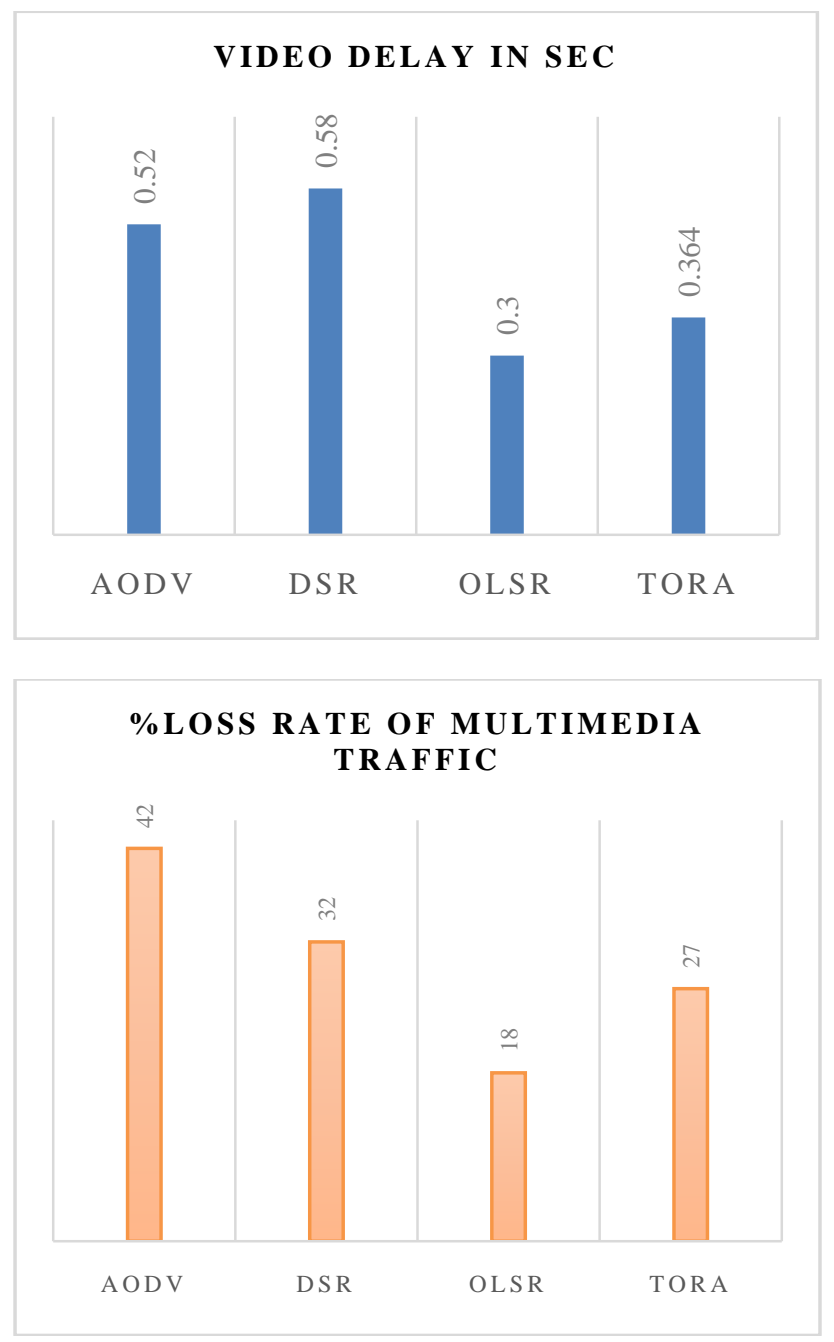

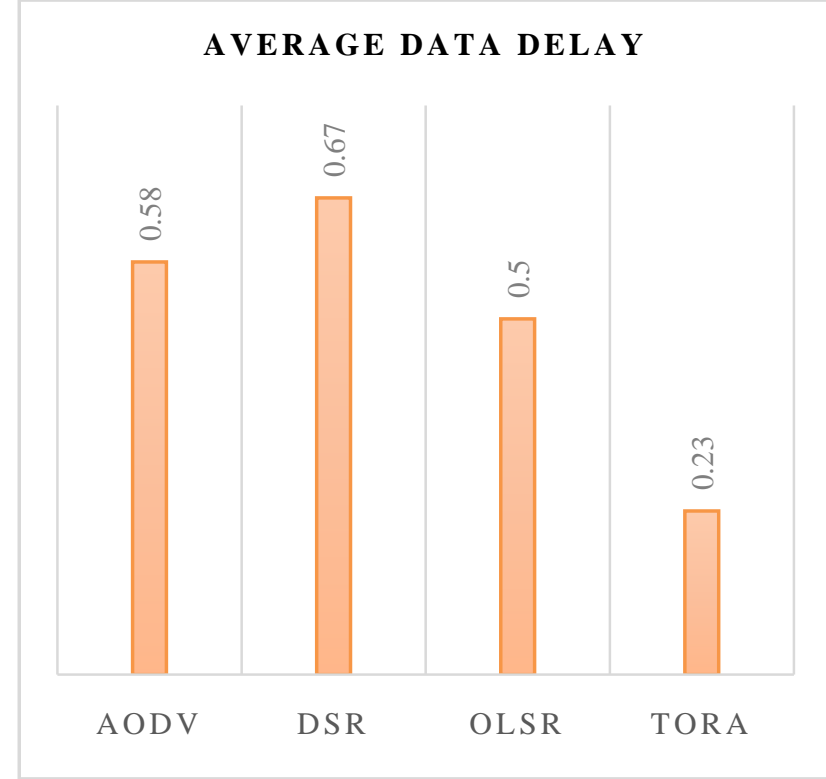

Fig 7: Network performance evaluation with high traffic load and mobility

Even if TORA was designed for high mobile networks. In the studied case, it is OLSR that gives high performances, in comparison with all the selected routing protocols. Even with no mobility support. We can conclude that the proactive nature of OLSR has served well this first ad hoc deployment.

\section{CONCLUSION}

We present in this paper, a comparative study of manets routing protocols, in an e-educational environment. Our study was already preceded by a comparative study of different 802.11 standards, in a preparative phase of deploying a large scale adhoc network to cover a whole university campus. Our motivation come from the fact that there is no ultimate comparative work of manets routing protocol who could generalize the fact of considering one routing protocol as better than another. So, in this project, it is imperative to make a choice of the most adequate protocol that fit manets for the proposed scenarisation. The study shows that for multimedia traffic OLSR routing protocol gives the best performance in comparison with AODV, TORA and DSR, for all the simulated scenarios. However, it is TORA who performs better considering data delays. No protocol is implementing a QoS mechanism for prioritizing a specific traffic. The nature of routing packets is different, and we can notice that TORA give better results while considering nodes mobility.

In this work we consider the same time for starting user's profiles. Our future work will take into consideration a specific scenarisation of generating traffic, which will be follow a real communication schema that will involve all university actors: professors, researchers, student etc. We plan to spread the study at a large scale.

\section{REFERENCES}

[1] Khiavi, M. Vajed, S. Jamali, and S. Jahanbakhsh Gudakahriz. "Performance comparison of AODV, DSDV, DSR and TORA routing protocols in MANETs." International Research Journal of Applied and Basic Sciences 3, no. 7 (2012): 1429-1436.

[2] Kuppusamy, P., K. Thirunavukkarasu, and B. Kalaavathi. "A study and comparison of OLSR, AODV and TORA routing protocols in ad hoc networks." In Electronics 
Computer Technology (ICECT), 2011 3rd International Conference on, vol. 5, pp. 143-147. IEEE, 2011.

[3] Nchimunya CHAAMWE1 and Langstone SHUMBA " eLearning Using Wireless Ad-Hoc Networks to Support Teaching and Learning in Rural Zambia " in Proceedings and report of the 7th UbuntuNet Alliance annual conference, pp 51-58, 2014.

[4] Michael Lauer and Michael Matthes. " ELAN: An ELearning Infrastructure for Ad-hoc Networks". Proceedings of the 8th ACM International Conference on Mobile Computing and Networking (MobiCom 2002), Poster Presentation, Atlanta, Georgia, USA, September 2002.

[5] Stieglitz, Stefan, Christoph Fuchß, O. Hillmann, and C. Lattemann. "Mobile learning by using ad hoc messaging network." In International Conference on Interactive Mobile and Computer Aided Learning, Amman, Jordan. 2007.

[6] F. Lakrami, N. Elkamoun. "Mobility and QoS Management in OLSR Routing Protocol." International Journal of Computer Networking, Wireless and Mobile Communications (IJCNWMC) 2.4 (2012): 2250-1568.
[7] C. Perkins, E. Belding-Royer, S. Das . "Ad hoc on-demand distance vector (AODV) routing". 2003.

[8] J. DB, M. DA, J. Broch "DSR: The dynamic source routing protocol for multi-hop wireless ad hoc networks". Ad hoc networking. Jan 8;5:139-72. 2001.

[9] F. Lakrami, N. Elkamoun, and M. El Kamili. "A Survey on QoS for OLSR Routing Protocol in MANETS." Advances in Ubiquitous Networking. Springer, Singapore, 2016. 287300 .

[10] A. Gupta, H. Sadawarti, A. Verma. "Performance analysis of AODV, DSR \& TORA routing protocols". International Journal of Engineering and Technology. 2010 Apr $1 ; 2(2): 226$.

[11] F. Lakrami, N. Elkamoun, O. Labouidya "Performance comparison of Wireless IEEE 802.11a,b, g and $\mathrm{n}$ used for Ad-Hoc Networks in an ELearning Classrooms Network" International Journal of Computer Science and Information Security, ISSN 1947-5500, Oct 2017.

[12] Modeler OP. Riverbed Technology. Inc. http://www. riverbed.com. 2016. 